\title{
153. Necessary Results for Computation of Cyclic Parts in Steinhaus Problem
}

\author{
By Kiyoshi IsÉKI
}

(Comm. by K. KunUGI, M.J.A., Dec. 12, 1960)

In my recent paper [1], we considered a problem of arithmetic. As the problem was stated in H. Steinhaus, Sto Zadan (in Polish), we call it Steinhaus problem for power $k$. We decided all cyclic parts of Steinhaus problem for power 3 (see may paper [1]). system:

Let $\alpha$ be a natural number, then $\alpha$ is expressed in the decimal

$$
\alpha=10^{n-1} a_{n}+10^{n-2} a_{n-1}+\cdots+10^{2} a_{3}+10 a_{2}+a_{1} .
$$

For $k=3$, let $\alpha_{1}$ be $a_{n}^{3}+a_{n-1}^{3}+\cdots+a_{3}^{3}+a_{2}^{3}+a_{1}^{3}$, then

$$
\begin{aligned}
\alpha-\alpha_{1}= & \left(10^{n-1}-a_{n}^{2}\right) a_{n}+\left(10^{n-2}-a_{n-1}^{2}\right) a_{n-1}+\cdots \\
& +\left(10^{2}-a_{3}^{2}\right) a_{3}+\left(10-a_{2}^{2}\right) a_{2}+\left(1-a_{1}^{2}\right) a_{1} .
\end{aligned}
$$

If $a_{1}=a_{2}=9$, then we have

$$
\begin{aligned}
& \left(1-a_{1}^{2}\right) a_{1}=-720, \\
& \left(10-a_{2}^{2}\right) a_{2}=-639,
\end{aligned}
$$

therefore, for any $a_{1}, a_{2}$, we have

$$
\left(1-a_{1}^{2}\right) a_{1}+\left(10-a_{2}^{2}\right) a_{2} \geq-1359 \text {. }
$$

Let $n \geq 4$, the function $\left(10^{n-1}-x^{2}\right) x$ of $x$ is an increasing function on $0 \leq x \leq 9$. Hence, $\left(10^{n-1}-x^{2}\right) x=1992$ for $n=4, x=2$, and we obtain

$$
\begin{aligned}
& \left(10^{n-1}-a_{n}^{2}\right) a_{n} \geq 1992, \\
& \left(10^{i-1}-a_{i}^{2}\right) a_{i} \geq 0 \quad(i=3,4, \cdots, n-1) .
\end{aligned}
$$

This implies

$$
\alpha-\alpha_{1} \geq 1992-1359=633
$$

and we have

$$
\alpha>\alpha-633 \geq \alpha_{1} .
$$

This means $\alpha>\alpha_{1}$ and the sequence $\alpha, \alpha_{1}, \alpha_{2}, \cdots$ for numbers greater than 2000 is strictly decreasing. Therefore we have a number less than 1999 for some term $\alpha_{n}$. Hence it is sufficient to find the cyclic parts of all numbers less than 1999.

Such an estimate is possible for every $k$.

For Steinhaus problem for $k=4$, from we have

$$
\alpha-\alpha_{1}=\left(10^{n-1}-a_{n}^{3}\right) a_{n}+\cdots+\left(10^{2}-a_{3}^{3}\right) a_{3}+\left(10-a_{2}^{3}\right) a_{2}+\left(1-a_{1}^{3}\right) a_{1},
$$

and, if $n=5, a_{n} \geq 2$, then

$$
\begin{gathered}
\left(10^{2}-a_{3}^{3}\right) a_{3}+\left(10-a_{2}^{3}\right) a_{2}+\left(1-a_{1}^{3}\right) a_{1} \\
\geq-(629 \times 9+719 \times 9+728 \times 9)=-18684, \\
\left(10^{i-1}-a_{i}^{3}\right) a_{i} \geq 0 \quad(i=4,5, \cdots, n-1)
\end{gathered}
$$

$$
\left(10^{4}-a_{5}^{3}\right) a_{5} \geq 19984 \text {. }
$$


This implies

and $\alpha-1300 \geq \alpha_{1}$.

$$
\alpha-\alpha_{1} \geq 1300,
$$

Then, for $k=4$ we must find cyclic parts of numbers less than 20000.

For $k=5$, the negative parts are greater than -226197 . If $n=6$, $a_{6} \geq 3$, then

Therefore we have

$$
\left(10^{5}-a_{6}^{4}\right) a_{6} \geq 299757
$$

$$
\alpha-73560>\alpha_{1} \text {, }
$$

From the argument above, for $k=5$ we must calculate the cyclic parts of numbers less than $3 \times 10^{5}$.

As we pointed out in my paper [1], for $k=3$, the cyclic parts we can decide by the table of cyclic parts from 1 to 999 .

Let us consider the numbers of the second column in the table. If the number $\alpha_{1}$ for $\alpha=a_{3} a_{2} a_{1}$ is larger than 1000 (for example, $\alpha_{1}=1073$ for $\alpha=179$ ), for $1 a_{3} a_{2} a_{1}\left(=1000+a_{3} a_{2} a_{1}\right)$, we have $\alpha_{1}=1+a_{3}^{3}+a_{2}^{3}+a_{1}^{3}$ (for 1179, we have 1074). If $\alpha_{1}$ contain the digit 0 , we need not consider the number $1 a_{3} a_{2} a_{1}$. Therefore, by the table in my paper [1], we need only consider: 1243 for 1189,1467 for 1299,1269 for 1389,1486 for 1399,1523 for 1499,1198 for 1579,1367 for 1589,1584 for 1599,1162 for 1669,1289 for 1679,1241 for 1688,1458 for 1689,1675 for 1699 , 1199 for 1778,1416 for 1779,1368 for 1788,1585 for 1789,1537 for 1888, 1754 for 1889, 1971 for 1899, and 2188 for 1999.

We can omit the calculation of $\alpha_{2}$, when it is less than 1000 . Then we have the following list:

$\begin{array}{ccccccc}\boldsymbol{\alpha} & \boldsymbol{\alpha}_{1} & \boldsymbol{\alpha}_{2} & \boldsymbol{\alpha}_{3} & & & \\ 1579 & 1198 & 1243 & 100 & 1 & & \\ 1679 & 1289 & 1250 & (125) & \mathbf{3 7 1} & & \\ 1778 & 1199 & 1460 & (146) & \mathbf{3 7 1} & & \\ 1899 & 1971 & 1074 & (147) & \mathbf{1 5 3} & & \\ 1999 & 2188 & 1033 & (133) & \mathbf{2 5 0} & \mathbf{2 1 7} & \mathbf{3 5 2}\end{array}$

Therefore, we have the complete solution of Steinhaus problem for $k=3$.

In our later paper, we shall use the results mentioned above to decide the cyclic parts for power 4,5 .

\section{Reference}

[1] K. Iséki: A problem of number theory, Proc. Japan Acad., 36, 578-583 (1960). 